\title{
Practical Kinetic Model for Hard Sphere Dynamics
}

\author{
James W. Dufty \\ Department of Physics, University of Florida, Gainesville, Florida 32611 \\ Andrés Santos \\ Departamento de Física, Universidad de Extremadura, E-06071 Badajoz, Spain \\ J. Javier Brey \\ Física Teórica, Universidad de Sevilla, E-41080 Sevilla, Spain
}

(Received 12 April 1996)

\begin{abstract}
The revised Enskog theory for a classical system of hard spheres provides a unified description of both fluid and crystal states over a wide range of densities and wavelengths. However, practical applications of this theory have been limited to fluid states near equilibrium. We propose here a simpler kinetic model that retains its essential physical and mathematical features, but which admits practical application to complex dynamical phenomena. This is illustrated by a calculation of the rheological properties of the fluid phase under shear flow. Good agreement is obtained in comparison with corresponding Monte Carlo simulation results. [S0031-9007(96)00873-3]
\end{abstract}

PACS numbers: 47.50.+d, 05.20.Dd, 05.60.+w, 83.50.Ax

The prototype realistic theory for dynamical properties of a many-body system is the Boltzmann kinetic theory. It has a strong basis in fundamental theory, and transport properties calculated from it for states near equilibrium are in excellent agreement with experimental studies for rarefied gases. Application of the Boltzmann kinetic equation for more complex states has been limited until relatively recently. During the past decade Monte Carlo simulation methods have been developed and applied with remarkable success [1]. Complementary analytic studies of states far from equilibrium have been obtained from simplified kinetic models based on the Boltzmann equation [2]. In several cases, comparisons of the kinetic model results with Monte Carlo simulation confirm the accuracy of the model theories even far from equilibrium [3].

The limitation of the Boltzmann equation to low density excludes its application to many of the most interesting phenomena: dense fluid transport, short wavelength dynamics, kinetics of freezing, crystal elasticity and transport, kinetics of metastable states, and dynamics of amorphous solid states. In spite of a great effort over the past 30 years, the only successful extension of the Boltzmann equation to high densities has been for the special case of hard spheres. This theory was developed by van Beijeren and Ernst [4] and is known as the revised Enskog theory (RET) because of its similarity to an earlier kinetic theory of Enskog. The RET is asymptotically exact at short times for a wide class of initial conditions and consequently has no a priori limitations on the density or space scale of the phenomena to be studied. In practice it extrapolates to long times as well. For example, it provides a good description of the dynamic structure factor as a function of wavelength and frequency, including both kinetic and hydrodynamic regimes, except at the highest densities near freezing [5]. While the RET was developed for the fluid phase it has been recognized recently that it admits the exact equilibrium crystal state as well [6]. Linear response studies of crystal states (elastic constants, transport coefficients) based on the RET are just beginning. In summary, the RET provides a unified description of fluid, crystal, and metastable states for the hard sphere system near and far from equilibrium, for length scales extending from hydrodynamics to less than the correlation length (hard sphere diameter). Thus, all of the interesting phenomena noted above can be addressed using the RET; we know of no other theory with a comparable scope.

Application of the RET has been limited almost exclusively to fluid states near equilibrium. During the past year, new Monte Carlo simulation methods similar to those for the Boltzmann equation have been developed for the RET [7,8]. However, as yet there has been no kinetic model for the RET to allow complementary analytic studies as in the Boltzmann case. The purpose here is to fill this gap by proposing a practical kinetic model that preserves all of the interesting qualitative features of the RET with the same domain of applicability. Together with the new Monte Carlo methods, both qualitative and quantitative aspects of high density complex phenomena, previously prohibitively difficult, can be addressed in detail. An example is provided here using the state of uniform shear flow, widely studied by molecular dynamics simulation [9] but with little theoretical support at the finite densities of interest.

The RET describes the time evolution of the one particle reduced distribution function, $f(\mathbf{r}, \mathbf{v}, t)$, for the position and velocity variables $(\mathbf{r}, \mathbf{v})$,

$$
\left(\partial_{t}+\mathbf{v} \cdot \nabla_{r}+m^{-1} \nabla_{v} \cdot \mathbf{F}\right) f=J[f]
$$


where $\mathbf{F}$ is an external force (possibly nonconservative) and $J[f]$ is the collision operator,

$$
\begin{aligned}
J[f](\mathbf{r}, \mathbf{v}, t)= & \sigma^{2} \int d \mathbf{v}_{1} \int d \Omega \Theta(\hat{\boldsymbol{\sigma}} \cdot \mathbf{g}) \hat{\boldsymbol{\sigma}} \cdot \mathbf{g} \\
& \times\left[\chi(\mathbf{r}, \mathbf{r}-\boldsymbol{\sigma} \mid n) f\left(\mathbf{r}, \mathbf{v}^{\prime}, t\right) f\left(\mathbf{r}-\boldsymbol{\sigma}, \mathbf{v}_{1}^{\prime}, t\right)\right. \\
& \left.-\chi(\mathbf{r}, \mathbf{r}+\boldsymbol{\sigma} \mid n) f(\mathbf{r}, \mathbf{v}, t) f\left(\mathbf{r}+\boldsymbol{\sigma}, \mathbf{v}_{1}, t\right)\right] .
\end{aligned}
$$

Here $\mathbf{g}=\mathbf{v}-\mathbf{v}_{1}, \mathbf{v}^{\prime}=\mathbf{v}-(\hat{\boldsymbol{\sigma}} \cdot \mathbf{g}) \hat{\boldsymbol{\sigma}}, \mathbf{v}_{1}^{\prime}=\mathbf{v}_{1}+(\hat{\boldsymbol{\sigma}}$. g) $\hat{\boldsymbol{\sigma}}, \boldsymbol{\sigma}$ is the hard sphere diameter, $\Theta(x)$ is the Heaviside step function, and $d \Omega$ denotes a solid angle integration for the unit vector $\hat{\boldsymbol{\sigma}}$. Finally, $\chi\left(\mathbf{r}, \mathbf{r}_{1} \mid n\right)$ is the local equilibrium pair correlation functional for a nonuniform system with density $n(\mathbf{r}, t)=\int d \mathbf{v} f(\mathbf{r}, \mathbf{v}, t)$.

It is clear that obtaining controlled approximate solutions to (1) is a formidable problem. In the spirit of kinetic models for the Boltzmann equation, we propose to replace (2) with a simpler form that preserves the essential properties of the RET. These properties include (1) local conservation laws for mass, energy, and momentum; (2) the exact equilibrium stationary state for both fluid and crystal phases; (3) an accurate description of structural effects on small space scales; and (4) a qualitatively correct prediction of density dependencies. To construct such a model from the RET, consider an orthonormal set of functions, $\psi_{\alpha}$, constructed from the set $\left\{1, \mathbf{v}, v^{2}\right\}$ with the scalar product

$$
\begin{aligned}
\left(\psi_{\alpha}, \psi_{\beta}\right) & \equiv \int d \mathbf{v} \phi_{\ell}(\mathbf{v}) \psi_{\alpha}^{*}(\mathbf{v}) \psi_{\beta}(\mathbf{v})=\delta_{\alpha \beta}, \\
\phi_{\ell}(\mathbf{v}) & =\left(\frac{\beta m}{2 \pi}\right)^{3 / 2} e^{-\beta m \tilde{v}^{2} / 2},
\end{aligned}
$$

where $\tilde{\mathbf{v}}=\mathbf{v}-\mathbf{U}$ is the velocity relative to the average local flow velocity $\mathbf{U}(\mathbf{r}, t)$ and $\left(k_{B} \beta\right)^{-1}=T(\mathbf{r}, t)$ is the temperature of the nonequilibrium state. A projection operator for this orthonormal set is given by $\mathcal{P} X=$ $\psi_{\alpha}(\mathbf{v}) \phi_{\ell}(\mathbf{v})\left(\psi_{\alpha}, \phi_{\ell}^{-1} X\right)$. To construct the kinetic model, the RET collision operator $J[f]$ first is decomposed into the two parts, $J[f]=(1-\mathcal{P}) J[f]+\mathcal{P} J[f]$. The second term on the right side represents contributions from collisions to the fluxes in the local conservation laws. It vanishes for the Boltzmann collision operator but is nonzero for the RET and gives the collisional transfer parts of the heat and momentum fluxes. It is necessary to retain this term in any approximate model in order to describe transport at moderate and high densities, and to preserve the correct stationary equilibrium distribution for the crystal phase. The first term on the right side of this decomposition does not contribute directly to the conservation laws and vanishes for both the crystal and fluid equilibrium states. The simplest choice is $(1-\mathcal{P}) J[f] \rightarrow-\nu(1-\mathcal{P}) f=-\nu\left(f-n \phi_{\ell}\right)$, where $\nu$ is a velocity independent collision frequency and use has been made of the property $\mathcal{P} f=n \phi_{\ell}$. The kinetic model we propose is therefore $J[f] \rightarrow-\nu(f-$ $\left.n \phi_{\ell}\right)+\mathcal{P} J[f]$. Further analysis of the last term on the right side shows it can be expressed in terms of the collisional transfer parts of the average heat flux, $\mathbf{q}^{c}(\mathbf{r}, t)$, and pressure tensor, $P_{i j}^{c}(\mathbf{r}, t)$, so that the model kinetic equation reads

$$
\begin{gathered}
\left(\partial_{t}+\mathbf{v} \cdot \nabla_{r}+m^{-1} \nabla_{v} \cdot \mathbf{F}\right) f=-\nu\left(f-n \phi_{\ell}\right) \\
-\beta \phi_{\ell}\left[\tilde{v}_{i} \partial_{j} P_{i j}^{c}+\frac{1}{3}\left(\beta m \tilde{v}^{2}-3\right)\left(\nabla \cdot \mathbf{q}^{c}+P_{i j}^{c} \partial_{i} U_{j}\right)\right] .
\end{gathered}
$$

The collisional transfer fluxes, $\mathbf{q}^{c}(\mathbf{r}, t)$ and $P_{i j}^{c}(\mathbf{r}, t)$, are the same nonlinear functionals of the distribution function $f$ as those obtained from the RET,

$$
\begin{aligned}
P_{i j}^{c}(\mathbf{r}, t)= & \frac{m}{2} \sigma^{3} \int_{0}^{1} d \lambda \int d \mathbf{v} \int d \mathbf{v}_{1} \int d \Omega \Theta(\hat{\boldsymbol{\sigma}} \cdot \mathbf{g})(\hat{\boldsymbol{\sigma}} \cdot \mathbf{g})^{2} \hat{\boldsymbol{\sigma}}_{i} \hat{\boldsymbol{\sigma}}_{j} \\
& \times \chi[\mathbf{r}-(1-\lambda) \boldsymbol{\sigma}, \mathbf{r}+\lambda \boldsymbol{\sigma} \mid n] f(\mathbf{r}-(1-\lambda) \boldsymbol{\sigma}, \mathbf{v}, t) f\left(\mathbf{r}+\lambda \boldsymbol{\sigma}, \mathbf{v}_{1}, t\right), \\
\mathbf{q}^{c}(\mathbf{r}, t)= & \frac{m}{2} \sigma^{3} \int_{0}^{1} d \lambda \int d \mathbf{v} \int d \mathbf{v}_{1} \int d \Omega \Theta(\hat{\boldsymbol{\sigma}} \cdot \mathbf{g})(\hat{\boldsymbol{\sigma}} \cdot \mathbf{g})^{2}(\tilde{\mathbf{G}} \cdot \hat{\boldsymbol{\sigma}}) \hat{\boldsymbol{\sigma}} \\
& \times \chi[\mathbf{r}-(1-\lambda) \boldsymbol{\sigma}, \mathbf{r}+\lambda \boldsymbol{\sigma} \mid n] f(\mathbf{r}-(1-\lambda) \boldsymbol{\sigma}, \mathbf{v}, t) f\left(\mathbf{r}+\lambda \boldsymbol{\sigma}, \mathbf{v}_{1}, t\right),
\end{aligned}
$$

where $\tilde{\mathbf{G}}=\frac{1}{2}\left(\mathbf{v}+\mathbf{v}_{1}\right)-\mathbf{U}$. These terms vanish in the low density limit so only the first term on the right side survives and the familiar Bhatnagar-Gross-Krook (BGK) kinetic model for the Boltzmann equation [10] is recovered. From the model (4), the problem of solving a kinetic theory with $\mathbf{r}, \mathbf{v}$, and $t$ as independent variables can be transformed to a problem of determining only fields depending on $\mathbf{r}$ and $t$. This is the key simplification that has allowed so much progress at the Boltzmann level using the low density kinetic model.

It is easily verified that (4) leads to the same local conservation laws for mass, energy, and momentum as those obtained from the RET. It is also verified that a stationary solution is given by $n_{s}(\mathbf{r}) \phi(v)$, where $\phi(v)$ is the Maxwellian distribution, in the absence of external forces. In this case $\mathbf{q}^{c}(\mathbf{r}, t)$ vanishes, the velocity integrals 
in (5) can be performed, and (4) reduces to

$$
\begin{aligned}
\mathbf{v} \cdot \nabla_{r} n_{s}(\mathbf{r})= & -\beta \tilde{v}_{i} \partial_{j} P_{i j}^{c} \\
= & \mathbf{v} \cdot \sigma^{2} \int d \Omega \hat{\boldsymbol{\sigma}} \chi\left(\mathbf{r}, \mathbf{r}-\boldsymbol{\sigma} \mid n_{s}\right) \\
& \times n_{s}(\mathbf{r}, t) n_{s}(\mathbf{r}-\boldsymbol{\sigma}) .
\end{aligned}
$$

The right side is a functional of $n_{s}(\mathbf{r})$ that is related to the gradient of the one particle direct correlation function, $c_{1}\left(\mathbf{r} \mid n_{s}\right)$, so that (7) can be simplified to $\nabla_{r} \ln n_{s}(\mathbf{r})=$ $\nabla_{r} c_{1}\left(\mathbf{r} \mid n_{s}\right)$. This is an identity for the special case of a uniform density (fluid phase). More generally, it is a well-known equation from density functional theory determining the equilibrium density for an inhomogenous system (crystal phase) [11]. Similar attempts to extend the BGK model to a description of two phases have occurred in the study of the lattice Boltzmann equation. This is the discrete (in space, time, and velocity) analog of the Boltzmann equation, for which the corresponding BGK kinetic model can be written. As in (4) an additional term like $P_{i j}^{c}(\mathbf{r}, t)$ has been added to describe both gas and fluid phases [12]. However, such equations are structurally much simpler than the RET, without energy conservation, the functional $\chi\left(\mathbf{r}, \mathbf{r}_{1} \mid n\right)$, or the broken symmetry crystal phase.

A first test of the kinetic model is its prediction of structural effects for states near equilibrium. The primary features of the linearized RET are a mean field force determined from the gradient of the two point direct correlation function, $c_{2}\left(\left|\mathbf{r}-\mathbf{r}^{\prime}\right|\right)$, and wavelength dependent matrix elements of the linearized collision operator in the subspace spanned by $\left\{1, \mathbf{v}, v^{2}\right\}$. These features are retained in the linearized form of (4) as well. To illustrate, the mean field force arises from linearization of the functional $\chi\left(\mathbf{r}, \mathbf{r}_{1} \mid n\right)$ in (5), leading to a term

$$
\begin{aligned}
& -\beta \sigma^{2} \int d \Omega \hat{\boldsymbol{\sigma}} n_{s}(\mathbf{r}+\boldsymbol{\sigma}) \frac{\delta}{\delta n_{s}\left(\mathbf{r}^{\prime}\right)}\left[\chi\left(\mathbf{r}, \mathbf{r}+\boldsymbol{\sigma} \mid n_{s}\right)\right] \\
= & -\beta \nabla\left[c_{2}\left(\left|\mathbf{r}-\mathbf{r}^{\prime}\right|\right)-\chi \Theta\left(\left|\mathbf{r}-\mathbf{r}^{\prime}\right|-\sigma\right)\right],
\end{aligned}
$$

where the right side defines the effective force. This term in the linear kinetic equation is responsible for features of the dynamic structure factor such as de Gennes narrowing. To test dynamical effects near equilibrium as well, the thermal conductivity, shear viscosity, and bulk viscosity have been computed from (4) by applying the Chapman-Enskog method to Navier-Stokes order [13]. The parameter $\nu$ is chosen to assure that the low density shear viscosity is the same as that from the Boltzmann equation, $\nu=n k_{B} T \chi / \eta_{B}$, where $\eta_{B}$ is the Boltzmann shear viscosity. As an example, the density dependence of the shear viscosity is found to be $\eta=\eta_{B} \chi^{-1}[1+$ $\left.(4 \pi / 15) \chi n^{*}+(64 \pi / 75)\left(\chi n^{*}\right)^{2}\right]$. This represents well the qualitative dependence of the RET (both are polynomials in $\chi n^{*}$ of degree two, where $n^{*} \equiv n \sigma^{3}$ ), with a maximum underestimate of about $25 \%$ at $n^{*}=0.54$.
Recently, a class of nonlinear transport coefficients associated with the pressure tensor for a fluid in shear flow has been calculated from the RET [14], and we have compared their density dependence with that obtained from the kinetic model for these more complex properties describing states farther from equilibrium. Again the method is a straightforward application of the ChapmanEnskog method to Burnett order, a formidable task for the RET but quite direct for the kinetic model. For example, the second viscometric function at small shear rates obtained from (4) is

$$
\begin{aligned}
\Psi_{2} & \equiv \lim _{a \rightarrow 0} a^{-2}\left(P_{z z}-P_{y y}\right) \\
& =-n k_{B} T \frac{128 \pi}{525}\left(\chi n^{*}\right)^{2}\left[1+\frac{4 \pi}{5} \chi n^{*}\right],
\end{aligned}
$$

where $a$ is the shear rate (in units of $\nu$ ). Again, the density dependence is found to be in qualitative agreement with that from the RET (both are polynomials in $\chi n^{*}$ of degree three). At the above density of $n^{*}=0.54$ the kinetic model overestimates $\Psi_{2}(a)$ by $50 \%$. These results indicate that the approximations leading to the kinetic model preserve the qualitative density dependence of the hard sphere fluid, even in a semiquantitative way.

We now consider an application of the kinetic model to a problem of nonlinear transport for which analytic studies using the RET have not been possible to date. The state considered is uniform shear flow. The macroscopic state is characterized by constant temperature and pressure, and a flow field $U_{x}=a y$. The shear rate $a$ is a control parameter that can be chosen to drive the system arbitrarily far from equilibrium. The viscous heating from this shear is compensated by an external nonconservative force. This state has been studied extensively via molecular dynamics computer simulation to illustrate rheological effects in simple atomic systems. In particular, it has been shown that the shear viscosity becomes a function of the shear rate $\eta(a)$ for states far from equilibrium. The only firm theoretical results for this function have been obtained from the Boltzmann equation for the special case of Maxwell molecules [15]; no results have been obtained from the RET as yet due to the complexity of the calculations involved. In contrast, it is straightforward to study this problem using the above kinetic model. The leading shear rate dependence of $\eta(a)$ is found from the Chapman-Enskog expansion to super Burnett order to be

$$
\begin{aligned}
\frac{\eta(a)}{\eta_{B}}= & \frac{\eta(0)}{\eta_{B}}-a^{2} \chi^{-1} \\
& \times\left[\frac{4}{3}+\frac{8 \pi}{15} \chi n^{*}+\frac{16 \pi}{75}\left(\frac{19}{7}+\frac{2 \pi}{9}\right)\left(\chi n^{*}\right)^{2}\right. \\
& \left.+\frac{512}{3375} \pi^{2}\left(\chi n^{*}\right)^{3}-\frac{4096}{39375} \pi^{2}\left(\chi n^{*}\right)^{4}\right] .
\end{aligned}
$$

At low and intermediate densities the coefficient of $a^{2}$ is negative, representing shear thinning. However, the term proportional to $\left(\chi n^{*}\right)^{4}$ is positive so there is a qualitative 


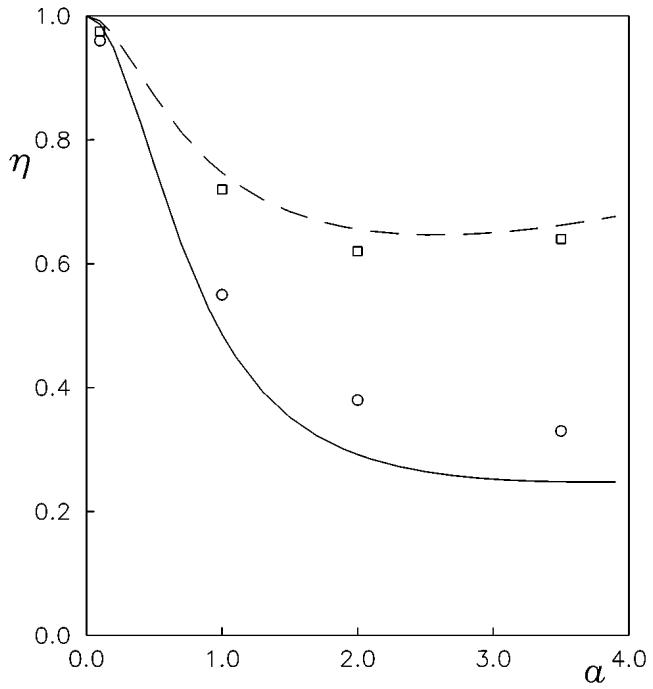

FIG. 1. Shear viscosity, $\eta(a) / \eta(0),(-, \bigcirc)$ and collisional transfer contribution, $\eta^{c}(a) / \eta^{c}(0),(--, \square)$ as functions of the shear rate in the uniform shear flow state at $n^{*}=0.2$. Lines are from the kinetic model; symbols are from Monte Carlo simulations of the RET.

change at higher densities from shear thinning to shear thickening. This is a remarkable new prediction of the kinetic model for the density dependence of the rheological properties. More generally, it is possible to show that for large shear rates $\eta(a) \sim a$ with a positive coefficient at all densities. Consequently, even at lower densities there is a crossover from shear thinning to shear thickening at sufficiently large shear rates.

The kinetic model also allows practical calculation of $\eta(a)$ for all shear rates. The term $P_{i j}^{c}$ in (4) is first treated as known and the exact solution is constructed. The resulting explicit distribution function is then used to calculate the kinetic and collisional parts of the shear viscosity in a self-consistent way. Recently, the new Monte Carlo methods developed for solving the RET [7] have been applied to this problem as well, so that a quantitative test of the model at finite density far from equilibrium is possible. Figure 1 shows the shear viscosity $\eta(a) / \eta(0)$ as a function of the shear rate at a moderate density of $n^{*}=0.2$ in comparison with the corresponding Monte Carlo results. Also shown is the collisional transfer contribution, which dominates at higher densities. The agreement is quite reasonable over the wide range of shear rates considered. It is possible also to see that both the kinetic model and simulation show the initial stages of shear thickening at the largest shear rates.

In summary, we return to the unique role of the RET as the only kinetic theory with the rich potential to address the entire scope of dynamical phenomena in ordered and disordered phases, at short and long wavelengths, large and small densities, near and far from equilibrium. It is not surprising that practical difficulties are encountered in the application of this equation. We have addressed this problem by providing a closely related description with the same potential, and demonstrated its practical application in several examples including some for which the RET is intractable. The quantitative discrepancies of the model are expected from the analogous studies of the Boltzmann equation for low density gas dynamics. In the latter case, this compromise has proved worthwhile as most information about the qualitative properties of transport far from equilibrium have been obtained from such models. We believe the kinetic model presented here will provide similar access to an even more interesting class of nonequilibrium phenomena at high densities heretofore unexplored. Also, the model can be extended to include the case of inelastic collisions [16] and applied to fast granular flows.

The research of J.W.D. was supported by NSF Grant No. PHY 9312723 and by a grant from the Division of Sponsored Research at the University of Florida. The research of J.J.B. and A.S. was partially supported by the DGICYT (Spain) through Grants No. PB95-534 and No. PB94-1021, respectively. The authors are grateful to J. Lutsko and J. M. Montanero for providing results prior to publication.

[1] G. Bird, Molecular Gas Dynamics and the Direct Simulation of Gas Flows (Clarendon Press, Oxford, 1994).

[2] For a recent review see J.W. Dufty, in Lectures on Thermodynamics and Statistical Mechanics, edited by $\mathrm{M}$. López de Haro and C. Varea (World Scientific, New Jersey, 1990), pp. 166-181.

[3] J. Gómez Ordóñez, J.J. Brey, and A. Santos, Phys. Rev. A 41, 810 (1990); J. M. Montanero, M. Alaoui, A. Santos, and V. Garzó, Phys. Rev E 49, 367 (1994); J. M. Montanero, A. Santos, and V. Garzó, Phys. Fluids (to be published).

[4] H. van Beijeren and M. H. Ernst, J. Stat. Phys. 21, 125 (1979).

[5] W.E. Alley, B. J. Alder, and S. Yip, Phys. Rev. A 27, 3174 (1983).

[6] T. R. Kirkpatrick, S.P. Das, M.H. Ernst, and J. Piasecki, J. Chem. Phys. 92, 3768 (1990).

[7] J. M. Montanero and A. Santos, Phys. Rev. E 53, 1269 (1996).

[8] F. J. Alexander, A. L. Garcia, and B. J. Alder, Phys. Rev. Lett. 74, 5212 (1995).

[9] Nonlinear Fluid Behavior, edited by H.J.M. Hanley (North-Holland, Amsterdam, 1983).

[10] C. Cercignani, Theory and Application of the Boltzmann Equation (Elsevier, New York, 1975).

[11] R. Evans, Adv. Phys. 28, 143 (1979).

[12] X. Shan and H. Chen, Phys. Rev. E 47, 1815 (1993); 49, 2941 (1993).

[13] J.H. Ferziger and H. G. Kaper, Mathematical Theory of Transport Processes in Gases (North-Holland, Amsterdam, 1972).

[14] J. Lutsko (unpublished).

[15] E. Ikenberry and C. Truesdell, J. Rat. Mech. Anal. 5, 1 (1956).

[16] J. J. Brey, J. W. Dufty, and A. Santos (to be published). 